\title{
Acute Alcohol Effects on Neuronal and Attentional Processing: Striatal Reward System and Inhibitory Sensory Interactions under Acute Ethanol Challenge
}

\author{
Mathias Schreckenberger*,', Rainer Amberg', Armin Scheurich ${ }^{3}$, Matthias Lochmann', Wolfgang Tichy', \\ André Klega', Thomas Siessmeier', Gerhard Gründer', Hans-Georg Buchholz', Christian Landvogt', \\ Jan Stauss', Klaus Mann ${ }^{3}$, Peter Bartenstein' and Reinhard Urban ${ }^{2}$ \\ 'Department of Nuclear Medicine, Johannes Gutenberg-University Mainz, Mainz, Germany; ${ }^{2}$ Institute of Legal Medicine, Johannes \\ Gutenberg-University Mainz, Mainz, Germany; ${ }^{3}$ Department of Psychiatry, Johannes Gutenberg-University Mainz, Mainz, Germany
}

\begin{abstract}
The acute influence of ethanol on cerebral activity induces complex psycho-physiological effects that are considerably more pronounced during acute ethanol influx than during maximal blood alcohol concentration (elimination phase). Despite the psychiatric and forensic relevance of these different ethanol effects, the underlying neuronal mechanisms are still unclear. In total, 20 male healthy volunteers were investigated each with three different experimental conditions in a randomized order using an intravenous ethanol challenge ( $40 \mathrm{~g}$ bolus infusion): during influx phase, elimination phase, and under placebo condition. During and after the ethanol (or placebo) infusion, neuropsychological testing of divided attention for visual and auditory stimuli was performed with subsequent I8-FDG PET acquisition. The PET data were analysed using SPM99. Ethanol influx and elimination phase showed focal activations in the bilateral striatum and frontal cortex and deactivations in the occipital cortex. The comparison of influx phase vs elimination phase revealed activations in the anterior cingulate and right prefrontal cortex, relevant deactivations were found in the left superior temporal cortex including Wernicke's area. Neuropsychological testing showed an attentional impairment under ethanol influx compared to ethanol elimination and placebo with an inverse correlation of the attentional performance for auditory stimuli to occipital activity and for visual stimuli to the left temporal (including auditory) cortex. Acute ethanol administration in healthy volunteers stimulates those striatal regions that are considered to have a particular relevance for alcohol craving ('reward system'). Modality specific reciprocal inhibition of sensory cortex activity seems to be relevant for attentional performance during acute alcohol impact.

Neuropsychopharmacology (2004) 29, I527-1537, advance online publication, 7 April 2004; doi: I0. I038/sj.npp. 1300453
\end{abstract}

Keywords: alcohol; ethanol; striatum; occipital; attention; imaging; PET; I8-FDG

\section{INTRODUCTION}

Although alcohol, having considerable medical and epidemiological relevance, is the most common 'drug' taken throughout the world, the neurophysiological processes underlying the psychotropic effects of acute ethanol intake are still unclear.

It is well known from clinical and forensic observations of acute ethanol effects that the complex psychophysiological changes during the acute cerebral ethanol uptake ('influx phase') is distinctive from those which occur after

\footnotetext{
*Correspondence: M Schreckenberger, Department of Nuclear Medicine, Johannes Gutenberg-University Mainz, Langenbeckstr. I, 5510। Mainz, Germany, Tel: + 496131172109 , Fax: + 49613117 2386, E-mail: schreckenberger@nuklear.klinik.uni-mainz.de

Received 8 October 2003; revised 18 February 2004; accepted 19 February 2004

Online publication: 4 March 2004 at http://www.acnp.org/citations/ Npp03040403464/default.pdf
}

the peak of blood alcohol concentration is reached ('elimination phase'), a phenomenon that was first described by Mellanby (1919). Early neuropsychological examinations under experimental alcohol intoxication revealed that performance deficits are more pronounced during the influx phase compared to the elimination phase, which led to the assumption that the maximal psychotropic alcohol effect precedes the maximal venous blood alcohol concentration (Bschor, 1952). Despite these early observations and the psychiatric and forensic importance of the different alcohol effects during influx and elimination, the neuronal mechanisms during the different phases of ethanol kinetics have not yet been thoroughly investigated.

The few functional imaging studies on acute alcohol effects published so far revealed neuronal activations in the right prefrontal cortex (Tiihonen et al, 1994), in the left temporal cortex and in left striatal regions (Wang et al, 2000) and the bilateral thalamus (Boecker et al, 1996), 
whereas decreases were found in the occipital cortex (Volkow et al, 1990; Wang et al, 2000) and in the bilateral cerebellum (Boecker et al, 1996).

First investigations combining positron emission tomography (PET) with [C-11]ethanol and magnetic resonance spectroscopy yielded that during the influx phase there is a high [C-11]ethanol uptake in the basal ganglia independently from regional cerebral blood flow (Amberg et al, 2002).

The majority of the investigations on acute alcohol impact on cerebral blood flow or cerebral glucose metabolism used oral alcohol administration in order to simulate 'physiological' uptake conditions (de Wit et al, 1990; Volkow et al, 1990; Schwartz et al, 1993; Boecker et al, 1996; Wang et al, 2000). The principle problems of this approach are (1) high interindividual variability of alcohol uptake by ingestion that impedes comparable arterial influx conditions and (2) impossibility to induce a fast arterial influx by means of 'normal' oral alcohol doses. Therefore, despite its 'unphysiological' character, an intravenous alcohol administration would enable a very similar arterial input and would provide standardized/comparable uptake conditions even measured over multiple subjects.

It was the purpose of this study to investigate the cerebral neuronal correlates of the different phases of ethanol kinetics compared to placebo by means of [F-18]fluorodeoxyglucose (18-FDG) PET.

\section{METHODS}

This study was carried out following the Helsinki Declaration and was approved by the local ethics committee, the Federal Health Administration (BfArM) and the radiation protection authorities (BfS).

\section{Subjects}

In total, 20 healthy male volunteers (range: 23-31 years) were included in this study. The subjects had no current or previous history of relevant physical illness, no current or past psychiatric disorders, no family history of major psychiatric disorder in first-degree relatives, and they were not regularly taking medication. All of the subjects, who were students from our medical school, were classified as 'social drinkers', who did not exceed a maximal mean alcohol intake of $30 \mathrm{~g}$ ethanol per day. Before entering the study, subjects were screened with a standard psychiatric interview (CIDI: Composite International Diagnostic Interview), a medical history, and a clinical examination including drug screening. All subjects gave written informed consent.

\section{Activation Protocol}

In a single-blinded study design, each subject underwent three PET scans with $150 \pm 20 \mathrm{MBq}$ [F-18]fluoro-deoxyglucose (18-FDG) and receiving $1 \times$ placebo and $2 \times$ ethanol infusion in a randomized order within a time interval of 4 weeks. All examinations were performed at the same time of day (all in the am, for the same subject time of injection within $1 \mathrm{~h}$ ) to minimize influences by circadian variability of cerebral glucose metabolism (Bartlett et al, 1988) or alcohol sensitivity.

On the examination day, subjects were asked to fast for at least $8 \mathrm{~h}$ before the PET study but had free access to water. The last time of alcohol consumption had to be at least $24 \mathrm{~h}$ ago. An indwelling cannula was placed in the veins of both lower arms for subsequent blood samplings. Placebo $(500 \mathrm{ml}$ $0.9 \%$ saline solution) and alcohol ( $40 \mathrm{~g}$ ethanol in $500 \mathrm{ml}$ $0.9 \%$ saline solution) were administered intravenously while the subjects were instructed to relax with eyes closed lying in a quiet and darkened room. Vital parameters were taken throughout the entire experiment. Neuropsychological testing was performed at different times before and during the ethanol challenge (see below) but not after the 18-FDG injection in order to avoid sensory or cognitive stimulation by the testing procedure during the relevant cerebral 18FDG uptake phase (0-30 min p.i.). Blood alcohol concentrations were measured before the onset of the infusion and 15 (at the end of the influx phase: see below), 30, 45 and $60 \mathrm{~min}$ after the start of the ethanol infusion.

\section{Ethanol Influx Condition}

Ethanol infusion was administered over 15 min with the 18FDG bolus at the beginning of the infusion. Neuropsychological testing was performed $5 \mathrm{~min}$ before ethanol infusion.

\section{Ethanol Elimination Condition}

Ethanol infusion was administered over 15 min with the 18FDG bolus $25 \mathrm{~min}$ after the end of the ethanol infusion. Neuropsychological testing was performed $5 \mathrm{~min}$ before ethanol infusion, $5 \mathrm{~min}$ after the beginning of the infusion (representing acute influx conditions), and $15 \mathrm{~min}$ after the end of the infusion (representing elimination conditions).

\section{Placebo Condition}

Placebo infusion was administered over 15 min with the 18FDG bolus at the beginning of the infusion. Neuropsychological testing was performed 5 min before placebo infusion.

\section{Neuropsychological Testing}

For the neuropsychological testing, two subtests of the computerized Testbattery for Attentional Performance (TAP 1.7; Zimmermann and Fimm, 2002) have been applied. The Divided Attention task uses visuo-spatial and auditory stimulation simultaneously.

The visual task was to detect squares made up by four crosses out of eight crosses that appear in 100 random configurations on the computer screen. The parallel acoustical task requires detecting irregularities in the alternating sequence of 200 high and low beeps. Outcome parameters are total reaction time (median), reaction time for visual and auditory stimuli (median) number of errors and omissions.

The subtest Flexibility requires focusing attention and shifting focus repeatedly between two sets of stimuli: numbers and letters. For 100 times, a letter and a number are presented simultaneously and randomly on the left or the right side of the fixation point on the computer screen. 
The test requires pressing the button either on the side where the number appears or where the letter appears. With each presentation the target changes from 'number' to 'letter'. Outcome parameters are reaction time (median), number of errors, and omissions.

Both subtests were processed repeatedly by every participant under the different experimental conditions. Training effects on test performance were controlled by the random application of the different conditions (placebo, elimination condition, influx condition).

\section{PET Examination and Image Reconstruction}

PET examinations were performed using an ECAT Exact PET Scanner (Siemens/CTI, Knoxville). The emission scan started $30 \mathrm{~min}$ after 18-FDG injection and continued for $20 \mathrm{~min}$ in a three-dimensional acquisition mode for one bed position (axial field of view of $16.2 \mathrm{~cm}$ ). Attenuation correction was performed using a computerized threshold limit routine to define an isodensity contour for the maximum cerebral activity/pixel. The exact position of these isodensity contours was controlled visually slice-byslice and eventually corrected manually. In all, 47 transversal slices (slice thickness $3.375 \mathrm{~mm}$ ) were reconstructed using filtered back projection with a $4 \mathrm{~mm}$ Hamming filter. The transaxial resolution was $6.0 \mathrm{~mm}$ FWHM (full-width at half-maximum).

\section{Absolute Quantification of Cerebral Glucose Metabolism}

Absolute glucose consumption rate (rMRGlu) was calculated for each pixel according to the autoradiography method after Sokoloff et al (1977) and Phelps et al (1979) using measured input function, tissue radioactivity concentration, and blood glucose concentration. For quantification of grey matter glucose metabolism, a set of rate constants $\mathrm{K} 1-\mathrm{k} 4$ and a lumped constant were used (Phelps et al, 1979; Reivich et al, 1985). Cortical Regions-of-Interest were manually drawn to calculate the mean cortical rMRGlu for each condition (influx, elimination, placebo).

\section{Statistical Analysis}

The differences of the mean absolute cortical glucose metabolism (rMRGlu) in terms of $\mu \mathrm{mol} / \mathrm{min} / 100 \mathrm{~g}$ during influx, elimination, and placebo were compared using the paired $t$-test. The further analysis of the PET data was performed by means of Statistical Parametric Mapping software (SPM 99, Wellcome Department of Cognitive Neurology, London). Due to the high intra- and interindividual variability of the absolute cerebral glucose utilization, normalized tissue activities (proportional scaling: see below) were used for the SPM analysis.

The PET images were realigned and stereotactically normalized into the standard anatomical space (Talairach and Tournoux, 1988) by means of linear and nonlinear transformation (Friston et al, 1995a). Subsequently, the normalized images were smoothed with a three-dimensional Gaussian filter using a $12 \mathrm{~mm}$ FWHM kernel, voxel size $2 \times 2 \times 2 \mathrm{~mm}^{3}$. The effect of the three different conditions (placebo/ethanol influx phase/ethanol elimination phase) on regional cerebral 18-FDG activity was estimated according to the general linear model (Friston et al, 1995b).

After proportional scaling of all PET scans to a mean global cerebral activity (Friston et al, 1990), $t$-statistical parametric maps (SPM $\{t\}$ ) were calculated. For the categorical comparisons (ethanol influx phase vs placebo, ethanol elimination phase $v s$ placebo, ethanol influx phase $v s$ ethanol elimination phase), the $t$-values of the scans were calculated on a voxel-by-voxel basis using a poled variance estimated from the whole-brain grey matter (Worsley et al, 1992). Since the resulting $t$-map is known to approximate closely to a standard Gaussian distribution (Worsley et al, 1992), the values were described as $Z$-scores. In order to determine a threshold for significant activation on the resulting $t$-map, the image smoothness was calculated (Friston et al, 1991), and a statistical threshold was estimated at a probability of $p<0.00001$. Resulting activation foci were considered significant, if they met a threshold of $p<0.05$, corrected for multiple comparisons across the whole-brain volume, based on the theory of random Gaussian fields (Lumer et al, 1998; Worsley et al, 1998).

The final step of the data analysis was the investigation of the functional correlation between the neuropsychological test performance and cortical metabolic activity. Those neuropsychological parameters (reaction time, number of errors, omissions), which were significantly reduced during ethanol influx or elimination, were correlated as an external covariate to the corresponding cortical metabolic data on a voxel-wise basis using a statistical threshold of $p<0.001$ (uncorrected) and a minimal cluster size $>80$ voxels (Bartenstein et al, 1997; Siebner et al, 2001).

As the image data were stereotactically normalized, maxima of the activation foci were reported with the respective stereotactic coordinates.

\section{RESULTS}

\section{Absolute Cortical Glucose Metabolism}

The mean cortical rMRGlu was significantly reduced $(p<0.0001)$ during the ethanol influx vs placebo (29.7 vs $39.2 \mu \mathrm{mol} / \mathrm{min} / 100 \mathrm{~g}$ ) and the ethanol elimination $v s$ placebo (29.2 vs $39.2 \mu \mathrm{mol} / \mathrm{min} / 100 \mathrm{~g}$ ), respectively. The very slight difference of the mean cortical rMRGlu between ethanol influx and ethanol elimination was not significant $(p=0.744)$.

\section{Ethanol Influx vs Placebo}

The categorical comparison of neuronal metabolic changes during ethanol influx $v s$ placebo revealed a significantly increased regional cerebral glucose metabolism above the given threshold of $p<0.05$ (corrected for multiple comparisons) in an extensive area over the bilateral striatum and including the anterior cingulate. Talairach coordinates of the voxels with the most significant increases were as follows: $x, y, z=-30,6,-2$ with $Z=6.68$. Additional metabolic increases were observed in the bilateral superior and medial frontal cortex $(x, y, z=-6,64,28, z=5.65)$ and in parts of the right midbrain $(x, y, z: 8,-22,-4, Z=4.96)$ and the left midbrain $(x, y, z:-10,-18,-12, Z=4.95)$. See Table 1a and Figure 1. 
Significant decreases of regional glucose metabolism were found in large areas of the bilateral primary and secondary visual cortex $(x, y, z: 20,-98,-2, Z=5.15)$, the left cuneus/ middle and superior occipital cortex $(x, y, z:-30,-94,20$,

Table I Increased (a) and Decreased (b) Regional Cerebral Glucose Metabolism Under Ethanol Influx Compared to Placebo $(p<0.05$, Corrected for Multiple Comparisons), See Figure I

\begin{tabular}{|c|c|c|c|c|}
\hline Area & $x$ & y & $\mathbf{z}$ & Z-score \\
\hline \multicolumn{5}{|c|}{ (a) Increased regional cerebral glucose metabolism } \\
\hline Bilateral striatum & -30 & 6 & -2 & 6.68 \\
\hline $\begin{array}{l}\text { Bilateral superior } \\
\text { and medial frontal } \\
\text { cortex }\end{array}$ & -6 & 64 & 28 & 5.65 \\
\hline Right midbrain & 8 & -22 & -4 & 4.96 \\
\hline Left midbrain & -10 & -18 & -12 & 4.96 \\
\hline \multicolumn{5}{|c|}{ (b) Decreased regional cerebral glucose metabolism } \\
\hline $\begin{array}{l}\text { Bilateral primary } \\
\text { visual cortex }\end{array}$ & 20 & -98 & -2 & 5.15 \\
\hline $\begin{array}{l}\text { Left cuneus/ } \\
\text { superior and } \\
\text { middle occipital } \\
\text { cortex }\end{array}$ & -30 & -94 & 20 & 5.84 \\
\hline $\begin{array}{l}\text { Left temporo- } \\
\text { occipital cortex }\end{array}$ & -54 & -70 & 0 & 5.09 \\
\hline
\end{tabular}

$Z=5.84)$ and the left temporo-occipital cortex $(x, y, z:-54$, $-70,0, Z=5.09$ ). See Table $1 \mathrm{~b}$ and Figure 1.

\section{Ethanol Elimination vs Placebo}

Subtraction analysis of the comparison ethanol elimination $v s$ placebo yielded similar findings as for the comparison of influx $v s$ placebo showing significant $(p<0.05$, corrected) increases of regional glucose metabolism in large areas of the bilateral striatum and parts of the anterior cingulate cortex. Talairach coordinates of the voxels with the most significant increases were as follows: $x, y, z:-36,2,-8$ with $Z=6.82$. There was also an increased metabolism in leftsided midbrain areas $(x, y, z:-16,-22,-14, Z=5.06)$. Table 2a.

Regional metabolic decreases were found (very similar to the comparison influx $v s$ placebo) in the bilateral primary and secondary visual cortex and adjacent cuneus $(x, y, z$ : $-4,-94,-4, Z=4.93)$ and in the bilateral parieto-occipital cortex (Brodman area 7) $(x, y, z: 2,-76,42, Z=5.12)$ (Table 2b).

\section{Ethanol Influx $v s$ Elimination}

The categorical comparison of the ethanol kinetic dependent changes revealed significant $(p<0.05$, corrected) regional metabolic increases during the influx phase in the anterior cingulate $(x, y, z:-6,50,0, z=5.41)$ and the

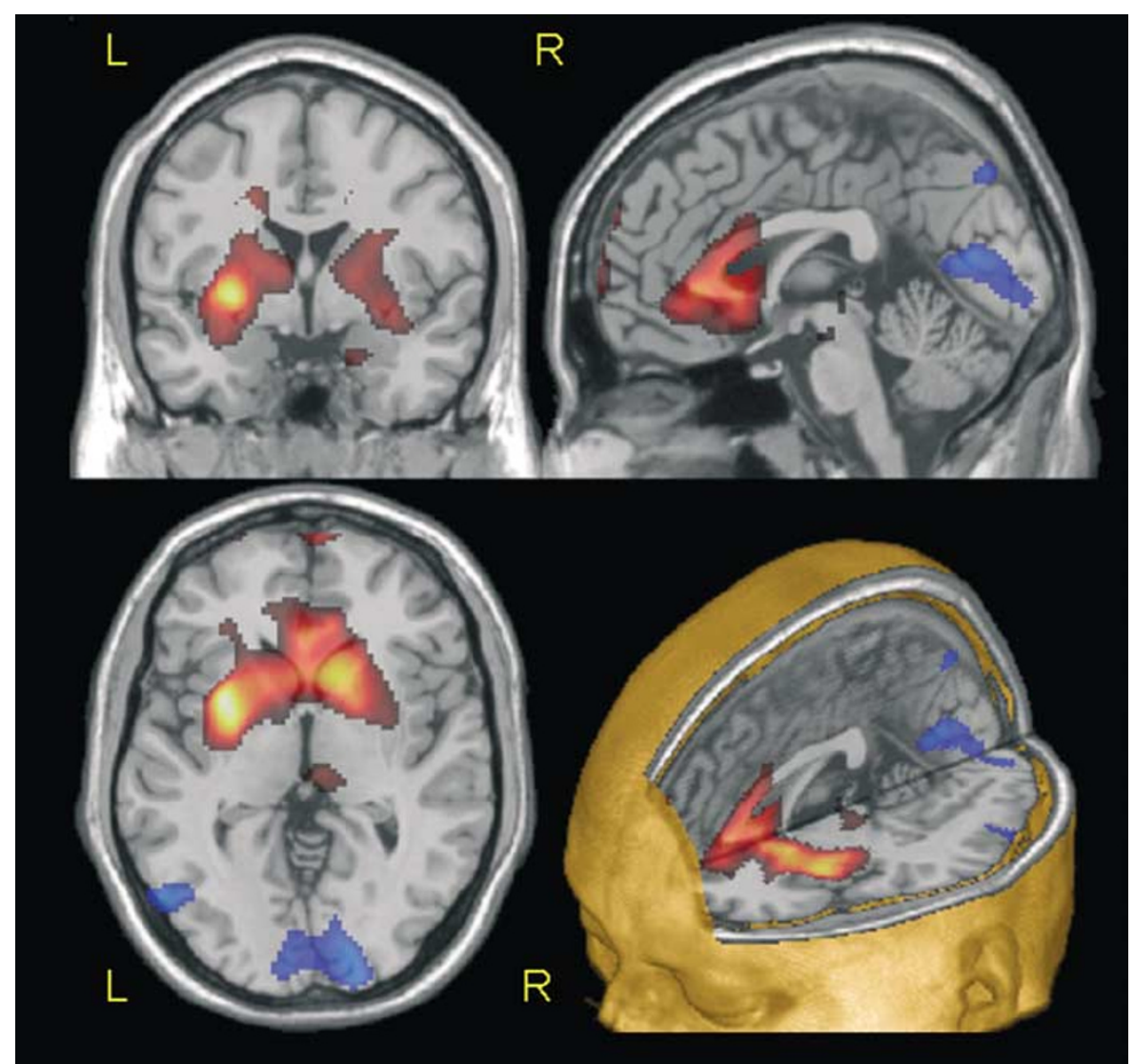

Figure I Comparison of ethanol influx vs placebo: activations (red 'hot metal') of the bilateral striatum, the anterior cingulate, parts of the superior and medial frontal cortex and of the midbrain. Deactivations (blue) of the visual cortex and the left temporo-occipital cortex. See Table Ia and b. 
Table 2 Increased (a) and Decreased (b) Regional Cerebral Glucose Metabolism Under Ethanol Elimination Compared to Placebo ( $p<0.05$, Corrected for Multiple Comparisons)

\begin{tabular}{|c|c|c|c|c|}
\hline Area & $x$ & y & $\mathbf{z}$ & Z-score \\
\hline \multicolumn{5}{|c|}{ (a) Increased regional cerebral glucose metabolism } \\
\hline $\begin{array}{l}\text { Bilateral } \\
\text { striatum }\end{array}$ & -36 & 2 & -8 & 6.82 \\
\hline Left midbrain & -16 & -22 & -14 & 5.06 \\
\hline \multicolumn{5}{|c|}{ (b) Decreased regional cerebral glucose metabolism } \\
\hline $\begin{array}{l}\text { Bilateral } \\
\text { primary and } \\
\text { secondary } \\
\text { visual cortex/ } \\
\text { cuneus }\end{array}$ & -4 & -94 & -4 & 4.93 \\
\hline $\begin{array}{l}\text { Bilateral } \\
\text { parieto- } \\
\text { occipital } \\
\text { cortex, BA } 7\end{array}$ & 2 & -76 & 42 & 5.12 \\
\hline
\end{tabular}

Table 3 Increased (a) and Decreased (b) Regional Cerebral Glucose Metabolism Under Ethanol Influx Compared to Ethanol Elimination ( $p<0.05$, Corrected for Multiple Comparisons), See Figure $2 \mathrm{a}$ and $\mathrm{b}$ respectively

\begin{tabular}{|c|c|c|c|c|}
\hline Area & $x$ & y & $\mathbf{z}$ & Z-score \\
\hline \multicolumn{5}{|c|}{ (a) Increased regional cerebral glucose metabolism } \\
\hline Anterior cingulated & -6 & 50 & 0 & 5.41 \\
\hline $\begin{array}{l}\text { Right prefrontal cortex } \\
\text { BA I I/47 }\end{array}$ & 44 & 30 & -12 & 5.64 \\
\hline \multicolumn{5}{|c|}{ (b) Decreased regional cerebral glucose metabolism } \\
\hline $\begin{array}{l}\text { Left posterior superior } \\
\text { temporal gyrus BA 13/22/41 }\end{array}$ & 44 & -26 & 4 & 5.21 \\
\hline $\begin{array}{l}\text { Left temporo-occipital cortex/ } \\
\text { left parahippocampal gyrus } \\
\text { BA I|/47 }\end{array}$ & -32 & -52 & -10 & 6.05 \\
\hline
\end{tabular}

right prefrontal cortex including the Brodman areas 11 and $47(x, y, z: 44,30,-12, Z=5.64)$. See Table $3 \mathrm{a}$ and Figure $2 \mathrm{a}$.

Decreases of regional glucose metabolism were found in the left posterior superior temporal gyrus $(x, y, z:-44,-26$, $4, Z=5.21)$ comprising parts of the Brodman areas 13, 22, and 41 . There was also a significantly reduced metabolism in larger areas of the left temporo-occipital regions including parts of the left parahippocampal gyrus: Brodman areas 11 and $47(x, y, z:-32,-52,-10, z=6.05)$. See Table $3 \mathrm{~b}$ and Figure $2 \mathrm{~b}$.

\section{Blood Alcohol Tests}

Blood alcohol concentrations for the influx condition taken at the end of the alcohol infusion (15 min p.i.) ranged from 0.7 to $2.14 \mathrm{ppt}$ (median $1.16 \mathrm{ppt}$ ). The blood alcohol concentrations measured $30 \mathrm{~min}$ p.i. ranged from 0.44 to $1.34 \mathrm{ppt}$ (median $0.73 \mathrm{ppt}$ ). For the elimination condition, blood alcohol concentrations taken $45 \mathrm{~min}$ p.i. ranged from 0.44 to $0.77 \mathrm{ppt}$ (median $0.6 \mathrm{ppt}$ ).

\section{Neuropsychological Testing}

Regarding neuropsychological performance in both subtests (Divided Attention and Flexibility), the subjects demonstrated only during the ethanol influx condition an impairment in terms of longer reaction times (see Table 4). In contrast, there were no differences in reaction times between ethanol elimination condition and placebo. In both subtests, error rates and number of omissions were highest during ethanol influx condition, but differences were not significant.

The task of Divided Attention revealed significant longer reaction times (median) for the ethanol influx condition relative to the elimination condition (repeated-measures ANOVA; post hoc analyses of pairwise differences: $p=0.004)$. Repeated-measures ANOVA only with reactions to the visual stimuli resulted in no significant difference between groups (influx condition relative to elimination condition: $p=0.794)$. Conversely, the reactions to auditory stimuli demonstrated a significantly longer reaction time (median) during the influx condition relative to the elimination condition (repeated-measures ANOVA; post hoc analyses of pairwise differences: $p=0.005$ ).

The Flexibility-task resulted in increased reaction time (median) for the influx condition relative to the elimination condition $(p=0.005)$. The difference in reaction time between influx condition and placebo did not reach the statistical threshold $(p=0.054)$.

\section{Correlation Analysis}

Since there were significantly longer reaction times in the Divided Attention test exclusively during the influx condition compared to the elimination condition and to placebo, the correlations between the reaction times and the regional cerebral glucose metabolism during the influx phase were investigated in order to detect those cortical areas, which were associated with attentional processes for auditory and visual stimuli, presented under acute ethanol influence.

For auditory stimuli, there was a significantly positive correlation between the median reaction time and the bilateral occipital cortex mainly the secondary visual cortex (right side: $x, y, z: 16,-100,-2 ; Z=3.93$; left side $x, y, z$ : $-34,-98,-12, Z=3.49)$ comprising of the Brodman areas 17 and 18 (see Table 5a and Figure 3a). In contrast, the median reaction time for visual stimuli was positively correlated to parts of the left temporal gyrus including auditory areas $(x, y, z:-6,-12,-6, Z=3.93)$ (see Table $5 \mathrm{~b}$ and Figure $3 b$ ). There were no negative correlations above the given statistical threshold.

\section{DISCUSSION}

The main findings of this study are that acute ethanol administration increases striatal neuronal activity and simultaneously decreases the activity of the primary and secondary visual cortex. The comparison of ethanol influx and ethanol elimination shows an activation in the anterior cingulate and in parts of the right prefrontal association 

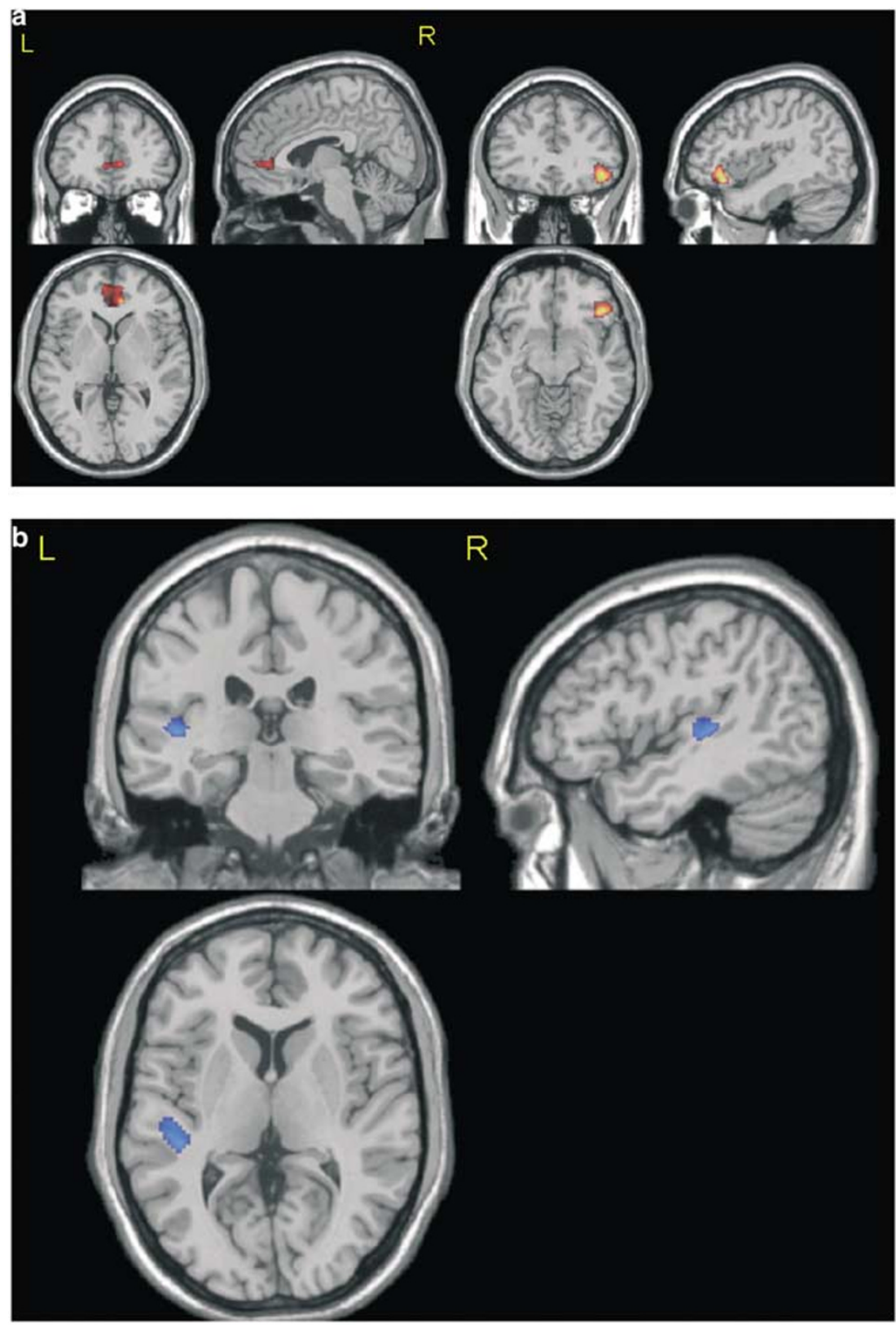

Figure 2 (a) Comparison of ethanol influx vs elimination: activations (red 'hot metal') of the anterior cingulate (upper row) and of the right prefrontal cortex (lower row). See Table 3a. (b) Comparison of ethanol influx vs elimination: deactivation (blue) of posterior parts of the left superior temporal gyrus including the Wernicke's area. See Table 3b.

cortex during the influx phase, whereas the activity of perisylvian areas (in particular of the Wernicke area) is decreased. The impaired attentional performance under ethanol influx is correlated to the bilateral occipital and left temporal activity depending on the sensory modality and indicating a reciprocal sensory inhibition for auditory and visual stimuli during acute ethanol impact.

\section{Methodical Considerations}

In this study design, we used functional imaging of cerebral neuronal activity by means of 18-FDG PET in order to assess cerebral glucose metabolism as an indicator of neuronal activity. Compared to [O-15]water PET for the measurement of cerebral blood flow with a temporal resolution of 10-30s (Silbersweig et al, 1993), 18-FDG shows a relatively slower temporal resolution of approximately $20 \mathrm{~min}$, which would indicate that 18 -FDG is more appropriate for the investigation of 'tonic' stimulation paradigms or pharmacological challenges (Volkow et al, 1990, 1999; Vollenweider et al, 1998; Gouzoulis-Mayfrank et al, 1999; Schreckenberger et al, 1999). Since the psychophysiological changes under acute alcohol influence are rather tonic, they are not expected to be adequately 


\begin{tabular}{|c|c|c|c|c|c|c|}
\hline & \multicolumn{2}{|c|}{ Influx condition } & \multicolumn{2}{|c|}{ Elimination condition } & \multicolumn{2}{|c|}{ Placebo } \\
\hline & $\mathbf{M}$ & SD & $\mathbf{M}$ & SD & $\mathbf{M}$ & SD \\
\hline Divided attention all stimuli: reaction time (median in ms) & 646* & 21 & 581 & 16 & 589 & 12 \\
\hline Divided attention: number of errors & 0.6 & 0.8 & 0.4 & 0.6 & 0.3 & 0.7 \\
\hline Divided attention: number of omissions & 1.3 & 2.3 & l. 1 & 1.7 & l. $\mid$ & 2.0 \\
\hline Divided attention visual stimuli: reaction time ( $\mathrm{md}$ in $\mathrm{ms}$ ) & 742 & 26 & 715 & 20 & 691 & 16 \\
\hline Divided attention auditory stimuli: reaction time (md in $\mathrm{ms}$ ) & $560^{*}$ & 20 & 501 & 15 & 489 & 17 \\
\hline Flexibility: reaction time (median in ms) & $557^{*}$ & 40 & 451 & 17 & 466 & 16 \\
\hline Flexibility: number of errors & 4.2 & 3.1 & 3.1 & 2.2 & 2.8 & 2.4 \\
\hline
\end{tabular}

Test performance (reaction time-median in ms and number of errors and omissions: mean (M) and standard deviation (SD)) during influx condition, elimination condition and placebo $(* p<0.01)$

Table 5 Cortical Areas Where Glucose Metabolism During Influx Condition was Positively Correlated to Median Reaction Time of Divided Attention Test for (a) Auditory Stimuli and (b) Visual Stimuli $(p<0.00$ I, Minimal Cluster Size $>80$ Voxels), See Figure $3 a$ and $b$ Respectively

\begin{tabular}{lcccc}
\hline Area & $\mathbf{x}$ & $\mathbf{y}$ & $\mathbf{z}$ & Z-score \\
\hline $\begin{array}{l}\text { (a) Auditory stimuli } \\
\text { Right middle occipital } \\
\text { gyrus/right cuneus BA 17/ }\end{array}$ & 16 & -100 & -2 & 3.93 \\
| 8 & & & & \\
Left inferior and middle & -34 & -98 & -12 & 3.49 \\
occipital gyrus/left cuneus & & & & \\
BA 17/I8 & & & & \\
& & & & \\
(b) Visual stimuli & & & & \\
Left middle and superior & -62 & & & \\
temporal gyrus & & & & \\
\hline
\end{tabular}

represented by an imaging procedure with very fast time resolution. Therefore, we regarded 18-FDG to be the more suitable tracer for the purpose of this study.

\section{Blood Alcohol Concentrations}

All blood alcohol concentrations at the first tests for the influx condition were found to be significantly higher than the calculated theoretical maximum according to the Widmark-Weyrich equation for the ethanol distribution in the human body, using a 0.7 factor and a minimal loss by diffusion of $10 \%$. This demonstrates still undistributed alcohol with a higher concentration in the blood pool compartment. In the influx condition, $30 \mathrm{~min}$ after 18-FDG administration the blood alcohol concentrations were either at the calculated maximal values or slightly higher, indicating the last minutes before the alcohol equilibrium. Together, both results prove the cerebral 18-FDG uptake under influx conditions. For the context of the elimination condition, all blood alcohol concentrations should have reached or surpassed the calculated maximal values. This was the case as demonstrated by the samples taken immediately before the 18 -FDG administration (45 min p.i.).

\section{Brain Metabolic Ethanol Effects}

The most important result of an ethanol induced increased striatal activity might be explained by a stimulation of the ventral striatal 'reward system' (Robbins and Everitt, 1996; Koob and Le Moal, 1997). All addictive drugs directly or indirectly influence the dopaminergic projections from the ventral tegmental area (VTA) to the forebrain, and here especially to the amygdala, the ventral pallidum, the prefrontal cortex, and particularly to the nucleus accumbens (NA) (Berke and Hyman, 2000; Wise and Rompre, 1989). The NA plays a crucial role in the reward system of the human brain (Robinson and Berridge, 1993; Koob and Le Moal, 1997; Everitt and Wolf, 2002). Lesions in this brain region abolish the dependence-inducing effects of many drugs of abuse, while stimulation of VTA neurons leads to decreased substance consumption (Di Chiara, 2000). However, the molecular events through which dopamine concentrations are increased in the NA markedly differ between the various drugs of abuse. Stimulants like amphetamine or cocaine increase dopamine release in the NA either directly or by blockade of the dopamine transporter (Reith et al, 1986; Pierce and Kalivas, 1997). Nicotine increases the firing rate of the dopaminergic neurons in the VTA via nicotinic acetylcholine receptors located on the cell bodies of these neurons (Mereu et al, 1987). Opiate receptors of the $\mu$-type are not directly situated on the dopaminergic mesocorticolimbic neurons, but on GABAergic interneurons and GABAergic afferents to the VTA. Stimulation of these receptors leads to inhibition of both tonic and phasic release of GABA, which is secondary followed by disinhibition of dopaminergic VTA neurons (Gysling and Wang, 1983; Dilts and Kalivas, 1989; Pontieri et al, 1996). On the other hand, $\mu$ opiate receptors are also found on GABAergic neurons of the NA, which receive the dopaminergic projections from the VTA neurons (Dilts and Kalivas, 1989).

The molecular events that lead to alcohol abuse and dependence are less well defined, which is mainly due to the multiplicity of alcohol actions in the human brain. In general, alcohol inhibits neuronal activity by an amplification 

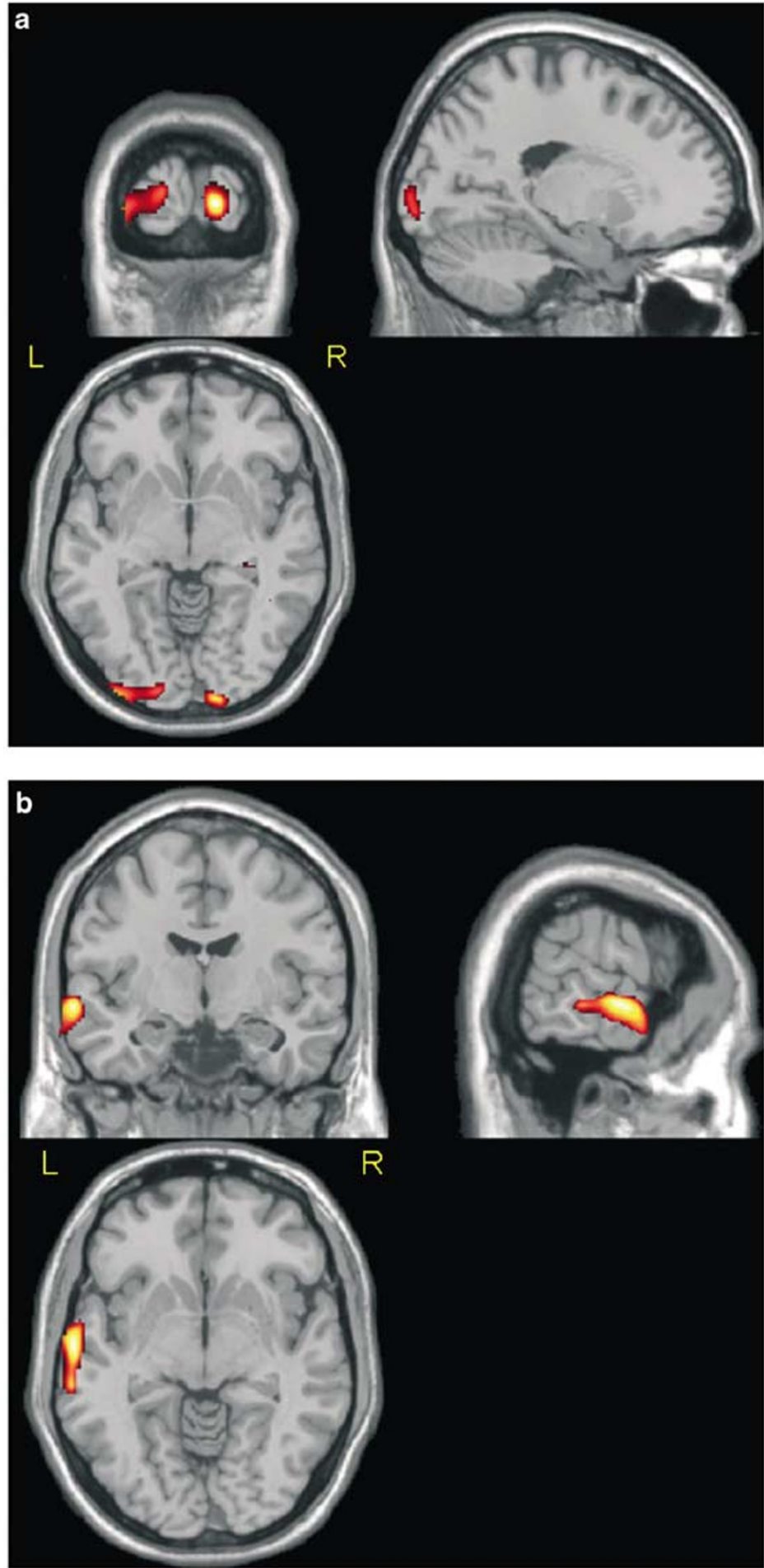

Figure 3 (a) Ethanol influx condition: Positive correlation (red 'hot metal') of the bilateral occipital (secondary visual) cortex activity to the median reaction time for auditory stimuli. See Table 5a. (b) Ethanol influx condition: Positive correlation (red 'hot metal') of the left middle and superior temporal (including auditory) cortex activity to the median reaction time for visual stimuli. See Table $5 b$.

of chloride currents and inhibition of glutamate-regulated sodium and calcium currents (Lovinger, 1997; Fadda and Rossetti, 1998). However, alcohol increases the firing rate of dopaminergic VTA neurons and releases dopamine in the NA (Yim et al, 1998; Bunney et al, 2000). These effects can be attributed to inhibition of the GABAergic interneurons of the VTA by alcohol. Since the alcohol-induced dopamine release in the NA can be antagonized by administration of an opiate receptor antagonist (Gonzales and Weiss, 1998), the inhibition of GABAergic VTA interneurons must at least partly be mediated by opioidergic afferents to these neurons. With regard to our study results, the observed ethanol-induced increase of striatal metabolic activity might reflect the 
disinhibition of dopaminergic VTA neurons (with subsequent dopamine release in the NA), which is most likely due to inhibition of GABAergic interneurons. On the other hand, it is well-known that inhibitory GABA receptors are most densely located in parieto-occipital cortices. Therefore, it is not surprising that the most prominent decreases in metabolic activity are observed in these cortical areas. The fact that these processes occur simultaneously underlines that they share the same biological basis.

The reduced activity of primary and secondary visual cortex, which is also observed by other investigators using oral ethanol administration, might represent the neuronal correlate of the well-described clinical phenomena of a narrowing of the visual field ('tunnel view') and the elevated visual perception threshold under acute alcohol impact. It could be shown by vestibular activation studies that a reciprocal inhibitory visual-vestibular interaction might protect visual perception from vestibular mismatches (Brandt et al, 1998). Therefore, further functional imaging studies on the role of alcohol-induced vestibular-visual correlations are mandatory to elucidate this aspect.

\section{Attentional Performance and Neuronal Activity}

The well-known fact from clinical observations of more heavily reduced psychophysiological performance during acute alcohol influx compared to a plateau or elimination has a particular psychiatric and forensic relevance. In this context, our finding of an impaired attentional test performance during ethanol influx compared to the ethanol elimination condition and placebo was not surprising but confirms the concept of maximal psychotropic alcohol effects before maximal alcohol uptake is reached. However, the cerebral correlates of this discrepancy between alcohol influx and elimination remained unclear up to now but were not adequately investigated by means of functional brain imaging.

The right prefrontal cortex activation might be associated with an associative thought loosening during acute alcohol influx. Remarkably, stimulation studies indicated activations of the medial frontal cortex and the anterior cingulate as a possible correlate of enhanced attentional processing of alcohol related stimuli (Bush et al, 2000). The finding of reduced activity in perisylvian areas/Wernicke's area during acute influx could be the correlate of an alcohol induced reduced word comprehension but which was not tested in our study design and, therefore, needs further investigation. As our results suggest, a perspective of the further line of research in the field of alcohol intoxication should focus on additional neuropsychological data (eg speech, memory). Neuropsychological testing should include tests of verbal comprehension (eg Verbal Concept Attainment Test, Bornstein and Leason, 1985); verbal fluency (Verbal Fluency Test) Benton and Hamsher, 1989 and tests of verbal memory (Auditory Verbal Learning Test, Rey, 1964; California Verbal Learning Test Delis et al, 1987).

One very interesting result of this study was that the attentional performance under ethanol influx showed reciprocal correlations to the metabolic activity of sensory cortex areas depending on the stimulus modality, which means that higher reduction of occipital (mainly secondary visual) cortical activity is associated with a better test performance for auditory stimuli whereas a higher reduction of left temporal (including auditory areas) cortical activity was correlated with higher attention to visual stimuli. This indicates that suppression of visual cortex activity increases the attentional performance for auditory stimuli and vice versa. Under alcohol induced perceptional changes, a reciprocal inhibitory auditory-visual interaction may provide a successful way to shift the dominant sensorial weight from one modality to the other as a compensatory mechanism. A similar inverse functional correlation in sensory activations could be shown for visual-vestibular stimulations of subjects without pharmacological challenge (Wenzel et al, 1996; Brandt et al, 1998) but was not described for auditory (Benedict et al, 1998; Pedersen et al, 2000, for review see Johnsrude et al, 2002) or visual (Kawashima et al, 1998; Bundesen et al, 2002) attentional processing in pharmacologically unaffected healthy subjects. In this context, the finding of a focal activation of the anterior cingulate during influx $v s$ elimination seems remarkable because the anterior cingulate is considered to have a relevant role for attentional processes in principle and is thought to encode in particular for selective attention ('selection for attention') (Demonet et al, 1993; Benedict et al, 1998). Thus, the finding of an increased anterior cingulate neuronal activity despite decreased neuropsychological performance might be the expression of an alcohol induced (insufficient) compensatory additional neuronal recruitment.

\section{CONCLUSION}

The present data indicate a close inverse relationship between striatal and occipital (visual) cortical activity under the acute influence of ethanol. Acute ethanol administration in healthy volunteers stimulates those striatal regions, which are considered to have a particular relevance for alcohol craving ('reward system'). A compensatory reciprocal inhibition of sensory cortex activity seems to be relevant for divided attentional performance during acute alcohol impact.

\section{ACKNOWLEDGEMENTS}

This study was supported by Deutsche Forschungsgemeinschaft (DFG): grants Be 454/4-2 and 4-3 and by 'Bund gegen Alkohol und Drogen im Straßenverkehr, Landessektion Rheinland-Pfalz'. Some of the results of this article form a part of the doctor's thesis of the coauthors Wolfgang Tichy and Andreas Klega at the Medical Faculty of the Johannes Gutenberg-University Mainz, Germany. Furthermore, we thank Mrs Jodi Neverve for her careful review of the manuscript.

\section{REFERENCES}

Amberg R, Fuermaier R, Hirt H, Urban R (2002). Regional ethanol absorption of the human brain during the influx phase measured by means of the Magnetic Resonance Spectroscopy (MRS) and Positron Emission Tomography (PET). Blutalkohol 39: 61-71.

Bartenstein P, Weindl A, Spiegel S, Boecker H, Wenzel R, CeballosBaumann A et al (1997). Central motor processing in 
Huntington's disease: a positron emission tomography study. Brain 120: 1553-1567.

Bartlett EJ, Brodie JD, Wolf AP, Christman DR, Laska E, Meissner M (1988). Reproducibility of cerebral glucose metabolic measurements in resting human subjects. J Cereb Blood Flow Metab 8: 502-512.

Benedict RH, Lockwood AH, Shucard JL, Shucard DW, Wack D, Murphy BW (1998). Functional neuroimaging of attention in the auditory modality. Neuroreport 9: 121-126.

Benton AL, Hamsher Kd (1989). Multilingual Aphasia Examination. AJA Associations: Iowa City.

Berke J, Hyman S (2000). Addiction, dopamine, and the molecular mechanisms of memory. Neuron 25: 515-532.

Boecker H, Wills AJ, Ceballos-Baumann A, Samuel M, Thompson PD, Findley LJ et al (1996). The effect of ethanol on alcoholresponsive essential tremor: a positron emission tomography study. Ann Neurol 39: 650-658.

Bornstein RA, Leason M (1985). Effects of localized lesions on the Verbal Concept Attainment Test. J Clin Exp Neuropsychol 7: 421-429.

Brandt T, Bartenstein P, Janek A, Dieterich M (1998). Reciprocal inhibitory visual-vestibular interaction. Visual motion stimulation deactivates the parieto-insular vestibular cortex. Brain 121: $1749-1758$.

Bschor F (1952). Beobachtungen über ein funktionales Prinzip bei der psychomotorischen Leistungsminderung in den verschiedenen Phasen der akuten Alkoholintoxikation. Dtsch $Z$ gerichtl Med 41: 273-276.

Bundesen C, Larsen A, Kyllingsbaek S, Paulson OB, Law I (2002). Attentional effects in the visual pathways: a whole-brain PET study. Exp Brain Res 147: 394-406.

Bunney E, Appel S, Brodie M (2000). Cocaine potentiates ethanolinduced excitation of dopaminergic reward neurons in the ventral tegmental area. J Pharmacol Exp Ther 293: 383-389.

Bush G, Luu P, Posner MI (2000). Cognitive and emotional influences in anterior cingulate cortex. Trends Cogn Sciences 4 215-222.

Delis DC, Kramer JH, Kaplan E, Ober BA (1987). California Verbal Learning Test: Adult Version. The Psychological Corporation: San Antonio, TX.

Demonet JF, Wise R, Frackowiak RSJ (1993). Language functions explored in normal subjects by positron emission tomography: a critical review. Hum Brain Mapping 1: 39-47.

Di Chiara G (2000). Role of dopamine in the behavioural actions of nicotine related to addiction. Eur J Pharmacol 393: 295-314.

Dilts RP, Kalivas PW (1989). Autoradiographic localization of muopioid and neurotensin receptors within the mesolimbic dopamine system. Brain Res 488: 311-327.

Everitt BJ, Wolf ME (2002). Psychomotor stimulant addiction: a neural systems perspective. J Neurosci 22: 3312-3320.

Fadda F, Rossetti Z (1998). Chronic ethanol consumption: from neuroadaptation to neurodegeneration. Progr Neurobiol 56: 385-431.

Friston KJ, Ashburner J, Frith CD, Poline JB, Heather JD, Frackowiak RSJ (1995a). Spatial registration and normalization of images. Hum Brain Mapping 3: 165-189.

Friston KJ, Frith CD, Liddle PF, Dolan RJ, Lammertsma AA, Frackowiak RS (1990). The relationship between global and local changes in PET scans. J Cereb Blood Flow Metab 10: 458-466.

Friston KJ, Frith CD, Liddle PF, Frackowiak RS (1991). Comparing functional (PET) images: the assessment of significant change. J Cereb Blood Flow Metab 11: 690-699.

Friston KJ, Holmes AP, Worsley KJ, Poline JB, Frith CD, Frackowiak RSJ (1995b). Statistical parametric maps in functional imaging: a general linear approach. Hum Brain Mapping 2: $189-210$

Gonzales RA, Weiss F (1998). Suppression of ethanol-reinforced behavior by naltrexone is associated with attenuation of the ethanol-induced increase in dialysate dopamine levels in the nucleus accumbens. J Neurosci 18: 10663-10671.

Gouzoulis-Mayfrank E, Schreckenberger M, Sabri O, Arning C, Thelen B, Spitzer M et al (1999). Neurometabolic effects of psilocybin, 3,4-methylenedioxy-ethylamphetamine (MDE) and d-methamphetamine in healthy volunteers. A double-blind, placebo-controlled PET study with [18F]FDG. Neuropsychopharmacology 20: 565-581.

Gysling K, Wang RY (1983). Morphine-induced activation of A10 dopamine neurons in the rat brain. Brain Res 277: 119-127.

Johnsrude IS, Giraud AL, Frackowiak RSJ (2002). Functional imaging of the auditory system: the use of positron emission tomograhy. Audiol Neurootol 7: 251-276.

Kawashima R, Satoh K, Goto R, Inoue K, Itoh M, Fukuda H (1998). The role of the left anterior temporal cortex for visual pattern discrimination - a PET study. Neuroreport 9: 1581-1586.

Koob GF, Le Moal M (1997). Drug abuse: hedonic homeostatic dysregulation. Science 278: 52-58.

Lovinger DM (1997). Alcohols and neurotransmitter gated ion channels: past, present and future. Naunyn-Schmiedeberg's Arch Pharmacol 356: 267-282.

Lumer ED, Friston KJ, Rees G (1998). Neural correlates of perceptual rivalry in the human brain. Science 280: 1930-1934.

Mellanby E (1919). British Medical Research Council, Special Report, Series No. 31.

Mereu G, Yoon K-WP, Boi V, Gessa GL, Nacs L, Westfall TC (1987). Preferential stimulation of ventral tegmental area dopaminergic neurons by nicotine. Eur J Pharmacol 141: 395-399.

Pedersen CB, Mirz F, Ovesen T, Ishizu K, Johannsen P, Madsen S et al (2000). Cortical centres underlying auditory temporal processing in humans: a PET study. Audiology 39: 30-37.

Phelps HE, Huang SC, Hoffmann EJ, Selin C, Sokoloff L, Kuhl DE (1979). Tomographic measurement of local cerebral glucose metabolic rate in humans with (F-18) 2-fluoro-2-deoxyglucose: validation of a method. Ann Neurol 6: 371-388.

Pierce RC, Kalivas PW (1997). A circuitry model of the expression of behavioral sensitization to amphetamine-like psychostimulants. Brain Res Rev 25: 192-216.

Pontieri FE, Tanda G, Orzi F, Di Chiara G (1996). Effects of nicotine on the nucleus accumbens and similarity to those of addictive drugs. Nature 382: 255-257.

Reith ME, Meisler BE, Sershen H, Lajtha A (1986). Structural requirements for cocaine congeners to interact with dopamine and serotonin uptake sites in mouse brain and to induce stereotyped behaviour. Biochem Pharmacol 35: 1123-1129.

Reivich M, Alavi A, Wolf AP, Fowler J, Russel J, Arne HC (1985). Glucose metabolic rate kinetic parameter determination in man: the lumped constant for (18-F) fluorodeoxyglucose and (11-C) deoxyglucose. J Cereb Blood Flow Metab 5: 179-192.

Rey A (1964). L' examen clinique en psychologie. Presses Universitaires de France: Paris.

Robbins TW, Everitt BJ (1996). Neurobehavioral mechanisms of reward and motivation. Curr Opin Neurobiol 6: 228-236.

Robinson TE, Berridge KC (1993). The neural basis of drug craving: an incentive-sensitization theory of addiction. Brain Res Brain Res Rev 18: 247-291.

Schreckenberger M, Gouzoulis-Mayfrank E, Sabri O, Arning C, Zimny M, Zeggel T et al (1999). 'Ecstasy'-induced changes of cerebral glucose metabolism and their correlation to acute psychopathology. An 18-FDG PET study. Eur J Nucl Med 26: $1572-1579$.

Schwartz JA, Speed NM, Gross MD, Lucey MR, Bazakis AM, Hariharan M et al (1993). Acute effects of alcohol administration on regional cerebral blood flow: the role of acetate. Alcohol Clin Exp Res 17: 1119-1123.

Siebner HR, Limmer C, Peinemann A, Bartenstein P, Drzezga A, Conrad B (2001). Brain correlates of fast and slow handwriting in 
humans: a PET-performance correlation analysis. Eur J Neurosci 14: 726-736.

Silbersweig DA, Stern E, Frith CD, Cahill C, Schnorr L, Grootoonk S et al (1993). Detection of 30 second cognitive activations in single subjects using a 3-D PET technique. J Cereb Blood Flow Metab 13(Suppl 1): S824.

Sokoloff L, Reivich M, Kennedy C, Des Rosiers MH, Patlak CS, Pettigrew KD (1977). The C-14-deoxyglucose method for the measurement of local cerebral glucose utilization: theory procedure and normal values in the conscious and anaesthesized albino rat. J Neurochem 28: 897-916.

Talairach J, Tournoux P (1988). A Co-planar Stereotaxic Atlas of the Human Brain. Stuttgart: Thieme.

Tiihonen J, Kuikka J, Hakola P, Paanila J, Airaksinen J, Eronen M et al (1994). Acute ethanol-induced changes in cerebral blood flow. Am J Psychiatry 151: 1505-1508.

Volkow ND, Hitzemann R, Wolf AP, Logan J, Fowler JS, Christmann D et al (1990). Acute effects of ethanol on regional brain glucose metabolism and transport. Psychiatry Res: Neuroimaging 35: 39-48.

Volkow ND, Wang GJ, Fowler JS, Hitzemann R, Angrist B, Gatley SJ et al (1999). Association of methylphenidate-induced craving with changes in right striato-orbitofrontal metabolism in cocaine abusers: implications in addiction. Am J Psychiatry 156: 19-26.

Vollenweider FX, Maguire RP, Leenders KL, Mathys K, Angst J (1998). Effects of high amphetamine dose on mood and cerebral glucose metabolism in normal volunteers using positron emission tomography (PET). Psychiatry Res 83: 149-162.

Wang GJ, Volkow ND, Franceschi D, Fowler JS, Thanos PK, Scherbaum $\mathrm{N}$ et al (2000). Regional brain metabolism during alcohol intoxication. Alcohol Clin Exp Res 24: 822-829.

Wenzel R, Bartenstein P, Dieterich M, Danek A, Weindl A, Minoshima $S$ et al (1996). Deactivation of human visual cortex during involuntary ocular oscillations: a PET activation study. Brain 119: 101-110.

Wise RA, Rompre PP (1989). Brain dopamine and reward. Annu Rev Psychol 40: 191-215.

de Wit H, Metz J, Wagner N, Cooper M (1990). Behavioral and subjective effects of ethanol: relationship to cerebral metabolism using PET. Alcohol Clin Exp Res 14: 482-489.

Worsley KJ, Cao J, Paus T, Petrides M, Evans AC (1998). Applications of random filed theory to functional connectivity. Hum Brain Mapping 6: 364-367.

Worsley KJ, Evans AC, Marrett S, Neelin P (1992). A threedimensional statistical analysis for CBF activation studies in human brain. J Cereb Blood Flow Metab 12: 900-918.

Yim HJ, Schallert T, Randall PK, Gonzales RH (1998). Comparison to local and systemic ethanol effects on extracellular dopamine concentration in rat nucleus accumbens by microdialysis. Alcohol Clin Exp Res 22: 367-374.

Zimmermann P, Fimm B (2002). Testbattery for Attentional Performance (TAP). Version 1.7. Psytest, Freiburg, Germany. 